\title{
Acousto-optic Effect in microstructured Polymer Fiber Bragg Gratings: Simulation and Experimental Overview
}

\author{
Carlos A.F. Marques, Lúcia Bilro, Lutful Kahn, Roberson A. Oliveira, David J. Webb, Member, IEEE, \\ and Rogério N. Nogueira, Member, IEEE
}

\begin{abstract}
A fine control of the microstructured polymer fiber Bragg grating spectrum properties, such as maximum reflected power and 3-dB bandwidth, through acousto-optic modulation is presented. For simulation purposes, the device is modelled as a single structure, comprising a silica horn and a fiber Bragg grating. For similar sized structures a good correlation between the numerical results and the experimental data is obtained, allowing the strain field to be completely characterized along the whole structure. It is also shown that the microstructured polymer fiber Bragg grating requires less effort from the piezoelectric actuator to produce modification in the grating spectrum when compared with a silica fiber Bragg grating. This technique has potential to be applied on tunable optical filters and tunable cavities for photonic applications.
\end{abstract}

Index Terms - Microstructured polymer optical fibers, Acousto-optic modulation, Fiber Bragg gratings.

\section{INTRODUCTION}

$\mathrm{P}$ OLYMER optical fibers (POFs) are starting to be considered a viable alternative to silica fibers (SF) in applications such as short distance transmissions [1, 2], sensing [3,4], Terahertz waveguides and filters [5], mainly due to their flexibility, larger core and simple connection. One of the major drawbacks pointed to POFs is the greater attenuation coefficient comparing to SF, a fact that limits their applications. However, recent studies show that the use of fluorinated polymers as core materials significantly reduces losses [6].

In-fiber Bragg gratings (FBGs) are pointed out as an interesting device for performing all-optical signal processing

Manuscript received November14, 2012; revised XXXX XX, 2012, XXX XX, 2012; accepted XXX XX, 2012.Date of publication XXX XX, 2012. The Fundação para a Ciência e a Tecnologia (FCT) Projects PTDC/EEATEL/114144/2009 and PTDC/EEA-TEL/122792/2010 are acknowledged. Carlos A. F. Marques and L. Bilro were supported by FCT under the SFRH/BD/70661/2010 and SFRH/BPD/78205/2011 scholarships).

Carlos A. F. Marques, Lúcia Bilro, Rogério N. Nogueira are with the Instituto de Telecomunicações, Pólo de Aveiro, 3810-193 Aveiro, Portugal (email: cmarques@av.it.pt, lucia.bilro@av.it.pt, rnogueira@av.it.pt).

Lutful Kahn, David J. Webb are with the Aston Institute of Photonic Technologies, Aston University, Birmingham, B4 7ET, UK (e-mail: khanmlh1@aston.ac.uk, d.j.webb@aston.ac.uk).

Roberson A. Oliveira is with the Volvo GTT - Advanced Technology \& Research, Av. Juscelino K. Oliveira, 2600, 81.260-900, Curitiba, Brazil (email: roberson.oliveira@volvo.com). and sensing, however most FBGs referenced in the literature are inscribed in SFs. On the other hand, for very short distance transmissions, POFs present advantages compared with conventional optical fibers, such as easy non-skilled handling and ruggedness. The scientific community has been investigating the possibility of inscribing FBGs in such fiber. A special type of POF is the microstructured POF (mPOF). Its geometry provides different properties compared to a step index fiber, such as an endlessly single-mode, air-guiding operation and the ability to expose the optical field of the guided modes to substances present in the holes. The first FBG inscribed in mPOF was obtained using a low-power helium cadmium $(\mathrm{HeCd})$ laser at $325 \mathrm{~nm}$ [7]. The resulting Bragg grating had a Bragg wavelength $\left(\lambda_{B}\right)$ of $1536 \mathrm{~nm}$ and a length of $10 \mathrm{~mm}$.

On the other hand, the acousto-optic (AO) effect is an important, fast and accurate mechanism that can be used to change and control several of the properties of Bragg gratings in SF and tapered fibers. It has been successfully applied in the design and construction of various low insertion loss alloptical processing devices such as a tunable optical notch filter [8], tunable mode coupler [9], complex Bragg grating writing [10], gain equalization filter [11], among others.

Concerning its mechanical properties, it is expected that the AO effect in mPOF would be more evident due to its lower acoustic impedance so improving the performance compared to the SF based devices. Therefore, this work demonstrates a fine control of the mPOF Bragg grating (mPOFBG) spectrum properties through the $\mathrm{AO}$ modulation. The interaction between an acoustic wave (AW) generated by longitudinal excitation and the written Bragg grating is presented. For simulation results, we apply the Finite Element Method (FEM) and Transfer Matrix Method (TMM) for designing an mPOFBG acousto-optic modulator (mPOFBG-AOM). The FEM approach allows the complete characterization of the strain field caused by the acoustic wave along the structure while the TMM is used to obtain the spectrum of the corresponding chirped grating. A similar theoretical approach is used to calculate the spectral response of an mPOFBG embedded in a host material system. Following the theoretical considerations, experimental results are also presented. 


\section{THEORETICAL BACKGROUND}

The FEM was introduced in the late 1950s in the aircraft industry [12]. The main advantages of the method are: the capability of modelling complex geometries, the consistent treatment of differential-type boundary conditions, and the possibility to be programmed in a flexible and general purpose format [13]. As the structure modelled here presents a variable shape along the longitudinal axis, the FEM is best suited to study the problem with the required accuracy. Fig. 1 shows the 1-D discretization of the FBG-AOM, whose main parts are the silica horn and fiber containing FBG. The structure is composed of $\mathrm{N}$ one-dimensional elements of length $\Delta \mathrm{z}=\mathrm{L}_{\mathrm{D}} / \mathrm{N}$ separated by nodes, where $\mathrm{L}_{\mathrm{D}}$ is the total length of the FBGAOM. Each element is associated with a value that represents the area of the structure at that section.

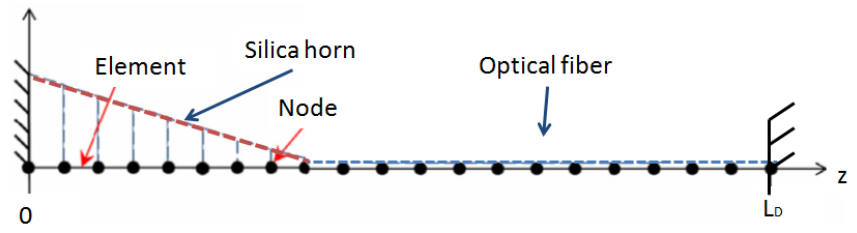

Fig. 1. Discretization of the FBG-AOM in linear elements. Each element has a characteristic function area.

The differential equation of motion that represents the acoustic wave propagation in the structure is known as:

$E \frac{\partial}{\partial z}\left(A(z) \frac{\partial u(z, t)}{\partial z}\right)-\rho A(z) \frac{\partial^{2} u(z, t)}{\partial t^{2}}=0$,

where $u$ is the axial displacement, which is dependent on the position $z$ and on the time $t$, and $d u / d z$ is the longitudinal strain $\varepsilon$. The term $A(z)$ accounts for the variable size of the structure along the $z$ axis. $E$ and $\rho$ are the Young modulus and density, assumed to be $72.5 \mathrm{GPa}$ and $2200 \mathrm{~kg} / \mathrm{m}^{3}$ for the silica and $3 \mathrm{GPa}$ and $1180 \mathrm{~kg} / \mathrm{m}^{3}$ for the polymer, respectively. One assumption established was to neglect the damping of the acoustic wave in the structure. The initial and boundary conditions are defined as:

$$
\left\{\begin{array}{c}
{\left[A E \frac{d u}{d z}\right]_{z=0}=P(t)=P_{D C}+P_{0} \exp (j w t)} \\
u\left(L_{D}, t\right)=0
\end{array}\right.
$$

The external excitation $P(t)$ is applied as the combination of a constant load $\left(P_{D C}\right)$ and a harmonic load of frequency $w$ and amplitude $P_{0}$ generated by the piezoelectric transducer (PZT). A classical linear approach for the finite elements is employed. After the one-dimensional discretization, the final matrix form of the problem is given by:

$M \ddot{u}+K u=P$,

where $u$ and $\ddot{u}$ represent the nodal displacement and acceleration vectors, respectively. The vector $P$ is the nodal generalized force and has a null value, except for the first component, associated with the node at $z=0$. In this expression, $M$ and $K$ are the mass and stiffness matrices of the structure, respectively, obtained by the superposition of the mass and stiffness matrix of each element, which are expressed as:

$$
\left[M_{i j}^{e}\right]=\frac{\rho A^{e} \Delta z}{2}\left[\begin{array}{ll}
1 & 0 \\
0 & 1
\end{array}\right]
$$

and

$$
\left[K_{i j}^{e}\right]=\frac{A^{e} E}{\Delta z}\left[\begin{array}{cc}
1 & -1 \\
-1 & 1
\end{array}\right],
$$

where superscript $e$ represents an element with particular properties. For the simulation, the applied load is assumed as being a concentrated point load at the base (larger diameter) of the silica horn. Considering the excitation in the form of $P=P_{D C}+P_{0} \exp (j w t)$ and assuming a linear system, the solution of the problem can be found by solving the equations:

$M \ddot{u}_{D C}+K u_{D C}=P_{D C}$,

$M \ddot{u}_{t}+K u_{t}=P_{0} \exp (j w t)$.

Eq. (6) represents the contribution of the component, $P_{D C}$, which is understood as a static pre-tension applied to the structure before the onset of the acoustic wave. Therefore, the acceleration is null and (6) can be reduced to:

$u_{D C}=\frac{1}{K} P_{D C}$.

Since the time dependent load generated by the piezoelectric is harmonic and $\ddot{u}_{t} \neq 0$, the solution for (7) has the form $u_{t}=u_{0} \exp (j w t)$. After replacing it in (7) one obtains:

$\left(-w^{2} M+K\right) u_{0}=P_{0}$

Note that $u_{0}$, the displacement vector solution, is highly dependent on the amplitude $P_{0}$ and frequency $w$ of the acoustic wave. Thus, the complete solution will be given by:

$u=u_{D C}+u_{0}$.

Once the displacement field is obtained, the strain field in each of the finite elements can be found by differentiation, as:

$\varepsilon^{e}=\frac{u^{e+1}-u^{e}}{\Delta z}$.

In this case, as the finite element is linear, $u^{e+1}$ and $u^{e}$ are the displacements in the local nodes $e+1$ and $e$, respectively.

On the other hand, the result for a uniform grating is an effective refractive index $\left(n_{\text {eff }}\right)$ perturbation in the core described by:

$\Delta n_{\text {eff }}(\mathrm{z})=\Delta \bar{n}_{\text {eff }}(\mathrm{z})\left\{1+v \cos \left[\frac{2 \pi}{\Lambda} \mathrm{z}\right]\right\}$

where $\Delta \bar{n}_{\text {eff }}$ is the average change of the effective refractive index, $v$ is the fringe visibility (assumed unitary in this work) and $\Lambda$ is the grating nominal pitch. As the grating imposes a dielectric perturbation to the waveguide, it forces the coupling between the propagating modes. The theory of coupled-modes $[14,15]$ is a useful and very well proven tool for describing this behavior. The set of coupled first-order differential equations used to describe the propagation is given by:

$\frac{d R}{d z}=j(\hat{\sigma} R(z)+\kappa S(z))$ 


$$
\frac{d S}{d z}=-j\left(\hat{\sigma} S(z)+\kappa^{*} R(z)\right)
$$

where $R(z)$ and $S(z)$ represent the propagating and counterpropagating modes, respectively; and the parameter $k$ is defined as

$$
\kappa=\frac{\pi}{\lambda} \Delta \bar{n}_{\mathrm{eff}}
$$

In these equations $\hat{\sigma}$ represents the general "dc" self-coupling coefficient, which is written as a sum of two terms:

$$
\hat{\sigma}=2 \pi n_{\text {eff }}\left(\frac{1}{\lambda}-\frac{1}{\lambda_{D}}\right)+\frac{2 \pi}{\lambda} \Delta \bar{n}_{\text {eff }} \text {. }
$$

In the first term called detuning, $\lambda_{D} \equiv 2 n_{\text {eff }} \Lambda$ is the "design wavelength" for a Bragg scattering within an infinitesimal variation of the effective index $\left(\Delta n_{e f f} \rightarrow 0\right)$, i.e., a grating that is infinitely weak.

This above described theory is applicable to uniform gratings, where the average refractive index change is constant. However, an acoustic wave will lead to a chirp in the grating, making its pitch non-uniform. Therefore, the reflection and transmission spectra from the two-mode coupling theory can be calculated by considering a piecewise approach, whereby the grating is divided into discrete uniform sections that are individually represented by a matrix. The solution is found by multiplying the matrices associated with each one of the sections. The characteristic equation is solved by equating to zero the matrix determinant. The resulting polynomial enables the determination of the eigenvalues.

The grating of length $L$ can be treated as a quadripole, as shown in Fig. 2. $R$ and $S$ represent the co-propagating and counter-propagating modes, respectively.

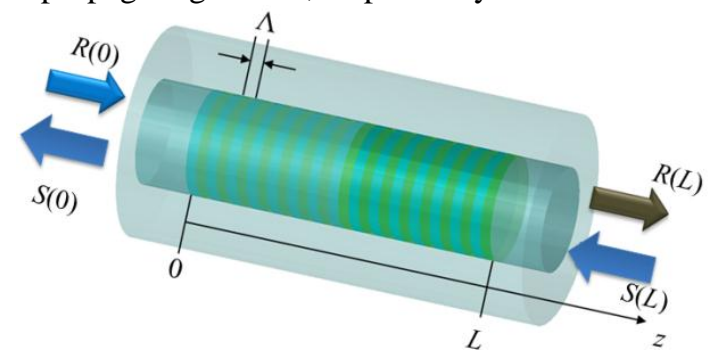

Fig. 2. Bragg grating in the core of an optical fiber.

For convenience, the amplitude $R(0)$ of the incident wave is normalized, in such a way that the maximum value is equal to the unit at the origin $(\mathrm{z}=0)$. Splitting the grating in $M$ uniform sections and defining $R_{i}$ and $S_{i}$ as amplitudes of the fields across the section $i$, the propagation is described by:

$\left[\begin{array}{c}R_{i} \\ S_{i}\end{array}\right]=T_{i}^{B}\left[\begin{array}{c}R_{i-1} \\ S_{i-1}\end{array}\right]$,

where $T_{i}^{B}$ is a $2 \times 2$ matrix given by

$$
T_{i}^{B}=\left[\begin{array}{cc}
\cosh \left(\gamma_{B} \Delta z\right)-j \frac{\hat{\sigma}}{\gamma_{B}} \sinh \left(\gamma_{B} \Delta z\right) & -j \frac{\kappa}{\gamma_{B}} \sinh \left(\gamma_{B} \Delta z\right) \\
j \frac{\kappa}{\gamma_{B}} \sinh \left(\gamma_{B} \Delta z\right) & \cosh \left(\gamma_{B} \Delta z\right)+j \frac{\hat{\sigma}}{\gamma_{B}} \sinh \left(\gamma_{B} \Delta z\right)
\end{array}\right],
$$

where $\Delta z$ is the length of the $\mathrm{i}^{\text {th }}$ uniform section and $\gamma_{B} \equiv \sqrt{\kappa^{2}-\hat{\sigma}^{2}}$. The coefficients $\hat{\sigma}$ and $k$ have local values at the $\mathrm{i}^{\text {th }}$ section. Since the matrices for each section are known, the application of the boundary conditions, $R(0)=1$ for $z \leq 0$ and $S(L)=0$ for $z>L$, causes the final equation to be:

$$
\left[\begin{array}{c}
R(0) \\
S(0)
\end{array}\right]=T^{B}\left[\begin{array}{c}
\mathrm{R}(\mathrm{L}) \\
\mathrm{S}(\mathrm{L})
\end{array}\right] \Rightarrow\left[\begin{array}{c}
1 \\
\mathrm{~S}(0)
\end{array}\right]=T^{B}\left[\begin{array}{c}
\mathrm{R}(\mathrm{L}) \\
0
\end{array}\right],
$$

where $T^{B}=\prod_{i=1}^{M} T_{i}^{\mathrm{B}}$ and writing $T_{i}^{\mathrm{B}}$ in the form of $T_{i}^{\mathrm{B}}=\left[\begin{array}{ll}\mathrm{T}_{11} & \mathrm{~T}_{12} \\ \mathrm{~T}_{21} & \mathrm{~T}_{22}\end{array}\right]$ and simplifying the Eq. (19), the result is:

$$
\left[\begin{array}{c}
1 \\
S(0)
\end{array}\right]=\left[\begin{array}{c}
T_{11} R(L) \\
T_{12} R(L)
\end{array}\right] .
$$

The $T_{11} \sim T_{22}$ terms represent the grating amplitude and phase response of the first segment. From (20) one concludes that $R(L)=\frac{1}{T_{11}}$. Therefore, the reflected amplitude for each wavelength can be found as

$$
\zeta(\lambda)=\frac{S(0)}{R(0)}=\frac{S(0)}{1}=\frac{T_{21}}{T_{11}}
$$

and the reflected power will be given by $r(\lambda)=|\zeta(\lambda)|^{2}$.

\section{Computational Simulation}

Fig. 3 shows the steps of the algorithm for the acousto-optic effect simulation. First, one is concerned with the input of the FBG-AOM dimensions, such as the radius and the length of the silica horn and the length of the Bragg grating. In the second step the FEM is used to model the device, whereby a desired load is applied to the base of the silica horn. The result is the strain field calculated along the structure. However, only the strain field in the FBG region is used as the input to the Transfer Matrix method. The shift of the design wavelength along the z-axis as a function of the strain field is assumed as:

$$
\lambda_{D}(z)=\lambda_{D 0}+\left(1+\left(1-p_{e}\right) \varepsilon(z)\right)
$$

where $p_{e}$ is the photoelastic coefficient and $\varepsilon(z)$ is the strain field calculated in the previous step through Eq. (11). It is important to note that this equation establishes the connection between the two methods. Finally, in the fourth step the TMM method gives the reflected and transmitted FBG spectra. The structure is designed using 26000 nodes/m. Each point along the structure corresponds to one element node. The quantity of nodes in the FBG region depends on its length. For example, for $L_{\text {Grating }}=10 \mathrm{~mm}$, there are 260 elements in the grating region, which is enough to obtain an accurate strain field for resonance frequencies in study. The number of sections in the TMM is chosen to be the same as the number of elements used in the FEM for the grating region. In the example, the discrete 
structure is excited with different acoustic wave frequencies of load amplitudes $P_{0}=1.5 \mathrm{~N}$ and $P_{D C}=0 \mathrm{~N}$, where $P_{0}$ will vary.

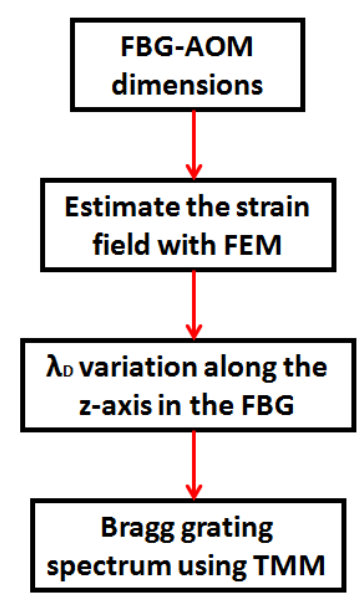

Fig. 3. Block diagram of the FBG-AOM simulation.

The mechanical properties of the mPOF can be achieved applying the theory of homogenization [16] or applying concepts of mixture rules of composite materials [17] used in this work. In order to adjust the FEM applied to a conventional FBG, the effective Young's modulus is calculated for the mPOFBG subtracting the equivalent amount of material on the homogeneous polymer fiber. Thus, the effective transversal area is given by:

$A_{e f f}=A-n \frac{\pi}{4}\left(d_{h}\right)^{2}$

where $n$ is the number of holes, $d_{h}$ is the diameter of each hole and $A$ is the transversal area of a circle with the same diameter of the fiber. This way, the effective Young's modulus for the $\mathrm{mPOF}$ is given by:

$E_{e f f}=E \frac{A_{e f f}}{A}$,

$\rho_{e f f}=\rho \frac{A_{e f f}}{A}$,

where $\rho$ is the density of the polymer. Knowing the effective area, the effective Young's modulus and the effective density, one can calculate the stiffness and mass matrices, used in the FEM analysis, treating the mPOFBG as a homogeneous conventional fiber [18]. The application of FEM to the mPOF model can be performed assuming geometrical symmetry for both main directions within transversal section. Here, we also consider the dimensions of the fiber used (SF or mPOF).

\section{Simulation Results AND Discussion}

When an acoustic wave is coupled into a fiber where an FBG is inscribed it generates a standing mechanical wave. A detailed study of the natural vibration modes of the mechanical structure can be found in literature [22] and the main results will be summarized briefly here for completeness. The FEM modal analysis using a commercial software (ANSYS) showed that: (i) most low frequency resonant modes generate flexural acoustical waves; (ii) longitudinal resonant modes are mostly observed in high frequency regimes (the acoustic longitudinal wavelength is small and the structure does not support the existence of flexural waves); (iii) at low frequency regimes, in an hybrid mode the flexural regime is dominant but there is also an interaction with a low frequency longitudinal acoustic wave. Flexural acoustic waves cause microbendings in the fiber and reduce the FBG reflectivity consequently. The effect of the longitudinal wave in the FBG modulation plans depends on its frequency namely the relationship between the acoustic wavelength and the FBG length. If the acoustic longitudinal wavelength is lower than the FGB length, the strain field transferred to the FBG has both compression and rarefaction and additional bands appear on both sides of the grating reflection spectrum. When the longitudinal wave is equal or longer than the FBG length the compression and/or rarefaction strain field will result in a shift of the Bragg wavelenght or even cause a linear variation in the grating period (chirp).

\section{A. Strain field behaviour}

Fig. 4 shows the modulator response (PZT is coupled to the horn-fiber system) in terms of fiber displacements, which is decomposed into axial ( $\mathrm{z}$ direction) and transversal displacements (xy direction). Although all analyzed acoustic modes have both components of the displacement field, the longitudinal acoustic waves are characterized by the larger displacements in $\mathrm{z}$ direction. The transversal displacements are due to lower amplitude flexural oscillations which are polarized in xy plane, transversally to the fiber axis. Also, at lower frequencies (flexural regime) the flexural acoustic waves are mostly characterized by the larger displacements in $\mathrm{xy}$ direction however, some displacements in $\mathrm{z}$ direction are observed.

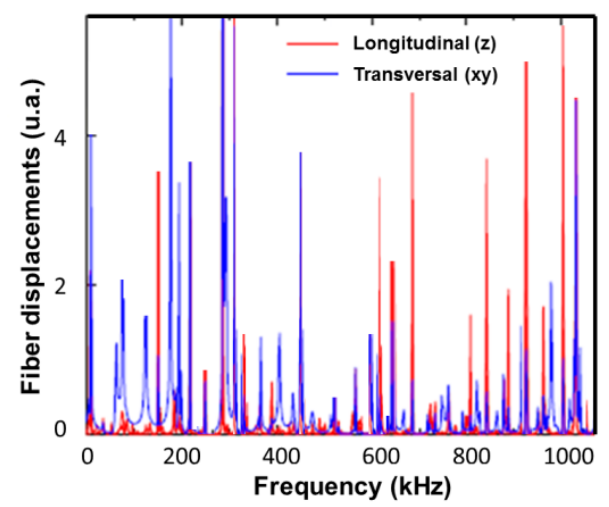

Fig. 4. AOM frequency response in terms of the fiber transversal and axial displacements.

A numerical comparison of the mechanical properties along mPOFBG-AOM and the similar structure with silica FBG (SFBG) is performed. Figs. $5(a)$ and $(b)$ detail the dimensions of the FBG-AOM used in the FEM model (similar to the experimental structure). A comparison of the strain field along the mPOFBG-AOM length and the similar structure with SFBG-AOM is shown in Figs. $5(c, e)-(d, f)$, when the grating length is $L_{\text {Grating }}=2 \mathrm{~mm}$ and $10 \mathrm{~mm}$, respectively. The strain field was achieved by FEM considering a sinusoidal excitation source applied on the base of the silica horn with resonance frequencies $\left(f_{R}\right)$ of $302 \mathrm{kHz}$ and $1 \mathrm{MHz}$, and load amplitudes 
$P_{0}=1.5 \mathrm{~N}$ and $P_{D C}=0 \mathrm{~N}$. According to the results presented in Fig. 4 and also considering a decomposition of the strain field, for $302 \mathrm{kHz}$ and $1 \mathrm{MHz}$ the strain field is presented by its transversal and longitudinal component, respectively. As can be seen in Fig. 5, the strain field amplitude is higher for the mPOFBG than for SFBG due to its smaller stiffness. It is also verified that the acoustic wavelength is shorter for the $\mathrm{mPOF}$, as expected. The behavior of the structure can be studied under the influence of parameters such as the frequency of the acoustic wave, the applied load or its dimensions. Using the dimensions given in Table 1 the following sections detail the behavior of the reflected grating spectrum as these parameters are varied.
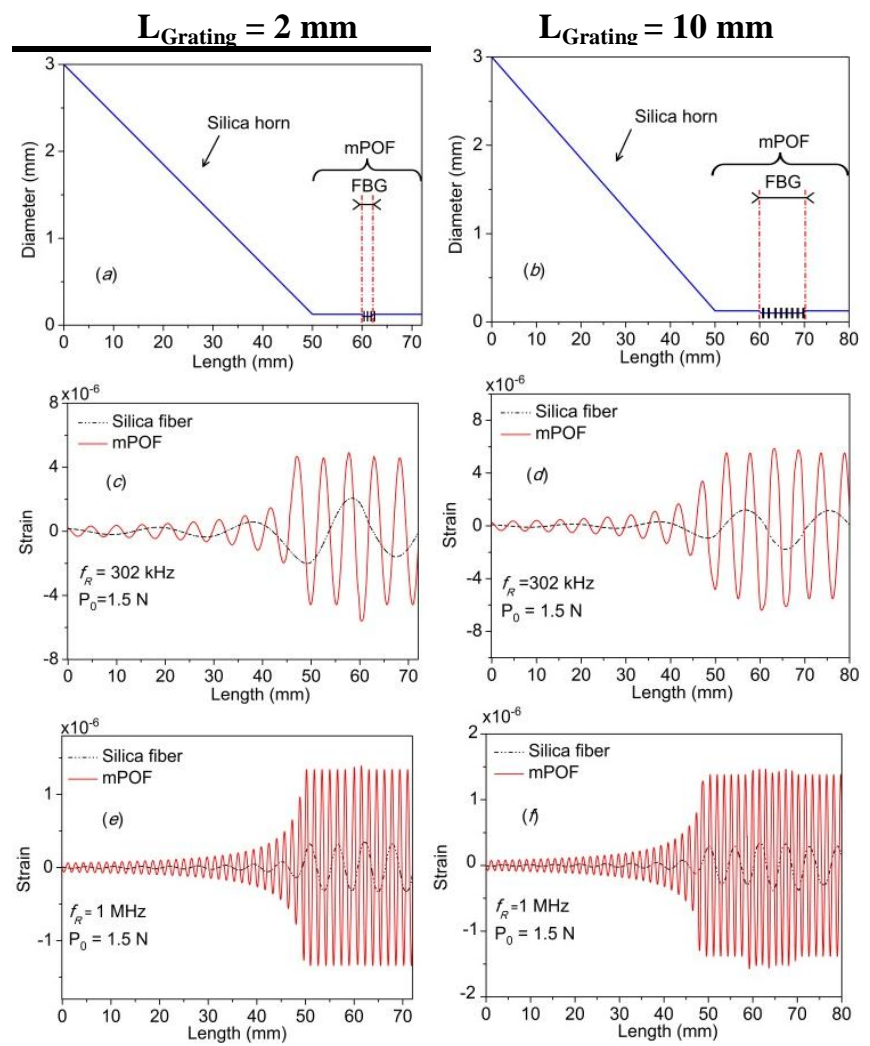

Fig. 5. Diameter of the AOM structure as function of the FBG length: (a) 2 $\mathrm{mm}$ and $(b) 10 \mathrm{~mm}$. Superposition of strain fields for mPOFBG and SFBG under the influence of the $\mathrm{AO}$ effect when grating length is $(c, e) 2 \mathrm{~mm}$ and $(d$, f) $10 \mathrm{~mm}$.

TABLE I. FBG LENGTH AND RESPECTIVE ACOUSTIC WAVE INTERACTION LENGTH OF THE HORN-FIBER

\begin{tabular}{c|c|c}
\hline FBG length $(\mathrm{mm})$ & 2 & 10 \\
\hline Interaction length: horn-fiber $(\mathrm{mm})$ & 72 & 80 \\
\hline
\end{tabular}

For a given device dimension the strain also varies with the PZT excitation frequency. The acousto-optic effect in the fiber will be enhanced when the excitation of the natural resonant modes of the silica horn-fiber structure are best matched to the frequencies delivered by the PZT. Previous results show that all resonant modes measured in the PZT are again observed in the combined structure, but with slight changes in frequency values because the anisotropic constants used in simulations did not include the PZT losses [22]. Those losses reduce the impedance-phase amplitude, which affects the localization of some resonances by the condition of minimum impedance and null phase. Fig. 6 shows strain variation as a function of the PZT excitation frequency for the dimensions detailed in Table 1 (in this case for $72 \mathrm{~mm}$ of interaction length). When a longer piece of $\mathrm{mPOF}$ is added on the set (the case of $80 \mathrm{~mm}$ interaction length - Table 1), it reduces the stiffness of the fiber, and a small shifting of the resonance frequencies range happens. Also, comparing these data with the results obtained in [21] for SF, the resonance frequencies are distinct for $\mathrm{mPOF}$, as expected, due to the different mechanical properties (mPOF and SF). The strain fields are much higher than for SF, with more than two orders of magnitude.

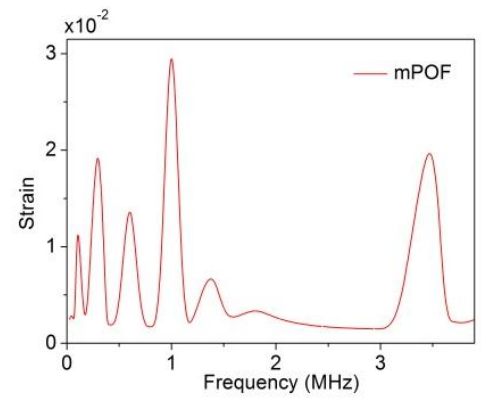

Fig. 6. Strain variation versus the PZT excitation frequency.

\section{B. Frequency and load variation}

Fig. 7 shows the grating reflection spectra calculated using the theory and computational simulation described in Sections II and III. By exciting the structure with acoustic waves of different frequencies the grating spectrum shows other characteristic wavelengths. We simulated the grating reflection spectra in $\mathrm{mPOF}$ for three different scenarios: (i) using flexural regime at $126 \mathrm{kHz}$ (Fig. 7 (a)); (ii) using longitudinal regime at $1 \mathrm{MHz}$ (Fig. $7($ b)) and $3.5 \mathrm{MHz}$ (Fig. 7 (c)); (iii) to obtain similar spectral shape in $\mathrm{SF}$ and $\mathrm{mPOF}$ (Fig. $6(d)$ ). For these scenarios the preload $\mathrm{P}_{\mathrm{DC}}$ is chosen to be null and the amplitude of the harmonic load $\mathrm{P}_{0}$ is changed. The FBG length is $L_{\text {Grating }}=2 \mathrm{~mm}$. In the first scenario the PZT actuator works in the bending regime at $126 \mathrm{kHz}$ as seen in Fig. 6 (a). It shall be noted that $P_{0}$ is much lower for mPOFBG due to smaller stiffness in comparison with the SFBG (inset of Fig. 7 (a)). As expected, a decrease of the grating reflectivity was achieved. In the second scenario the PZT actuator works in the stretching and compression regime, with symmetric displacement amplitude as seen in Fig. 7 (b) and (c). Fig. 7 (b) shows also the grating reflection spectrum when $P_{0}=0 \mathrm{~N}$ is applied at $1 \mathrm{MHz}$ showing one central peak, $\lambda_{B O}$. Applying load, side bands appear and if $P_{0}$ is increased, more side bands appear but the central band is attenuated. The wavelength difference between the central band and the side bands is estimated as $\Delta \lambda=\lambda_{\mathrm{B} 2}-\lambda_{\mathrm{B} 0}=\lambda_{\mathrm{B} 1}-\lambda_{\mathrm{B} 0}=\lambda_{\mathrm{B} 3}-\lambda_{\mathrm{B} 1}=\lambda_{\mathrm{B} 4}-\lambda_{\mathrm{B} 2}$ $=1.08 \mathrm{~nm}$. The same difference is obtained between consecutive side bands. Fig. 7 (c) shows a similar behavior with a higher resonance frequency. The figure also shows a greater difference between the side bands due to the frequency increase. To obtain similar spectral shape in the SF and $\mathrm{mPOF}$, it was necessary to apply a lower resonance frequency as well as lower PZT load to the mPOF as shown in the Fig. 7 (d). It shows that the mPOFBG requires less effort from the PZT actuator. 

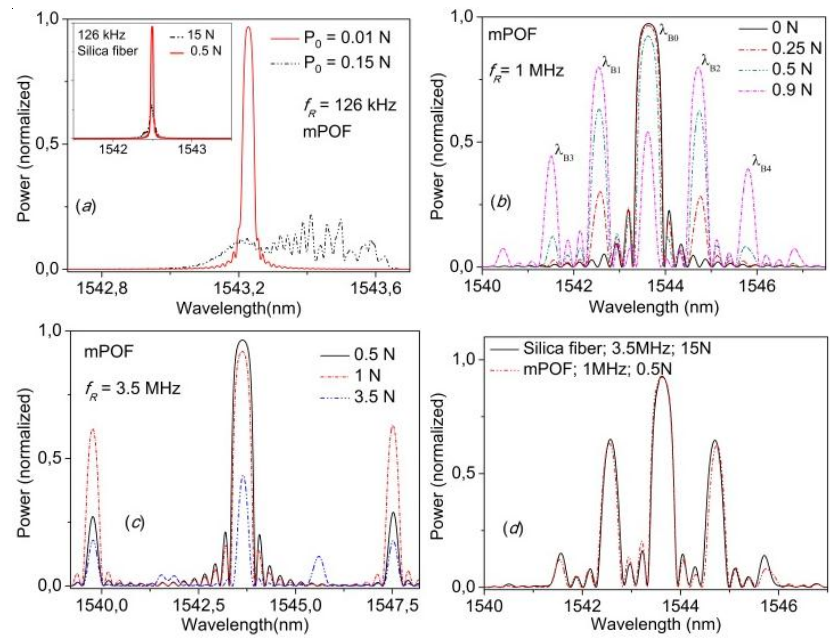

Fig. 7. Reflected spectra when excitations are applied with frequency at $(a)$ $126 \mathrm{kHz},(b) 1 \mathrm{MHz}$ and (c) $3.5 \mathrm{MHz}$. (d) Similar spectral shape SF and $\mathrm{mPOF}$. The FBG length is $2 \mathrm{~mm}$.

\section{Influence of the dimensions}

The influence of the horn dimensions on the strain observed along the structure allows the optimization of each of its parts. Applying different resonance frequencies and PZT loads, the strain field seen by the grating can be modified through changes in the dimensions of the silica horn. Fig. 6 shows the strain behavior as a function of the diameter and of the length of the silica horn for SF and mPOF. A modification of the horn dimensions causes changes in the resonance frequencies of the device. Numerical results show that an increase of the silica horn base diameter causes an increase in the stiffness and mass of the system, leading to a decrease of the displacement and strain fields along the FBG (Figs. 8 (a) and (b)). Fig. 8 (c) shows the results when a low amplitude load of $P_{0}=0.05 \mathrm{~N}$ is applied and we concluded that the strain fields are higher for $\mathrm{mPOF}$, regardless of the applied voltage and resonance frequency. By simulation results, the silica horn with $1 \mathrm{~mm}$ of base diameter presents the best result in terms of strain field. On the other hand, a change of the silica horn length does not cause significant variations in these fields, as shown in Fig. 8 (d), and, once more, the mPOF strain fields are much higher than for SF regardless the resonance frequency and FBG length. The strain field is also higher (more than one order of magnitude in relation to Fig. 8 (a)) when long FBGs are used as shown in Fig. 8 (b).
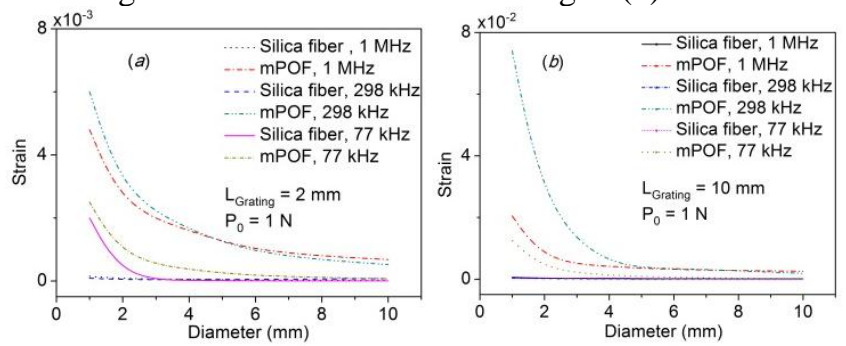
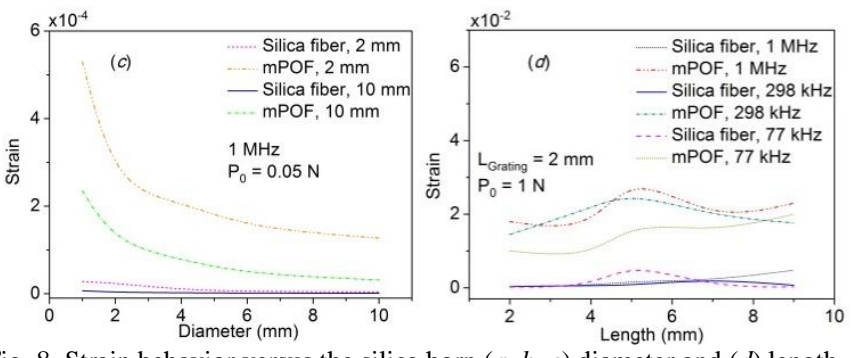

Fig. 8. Strain behavior versus the silica horn $(a, b, c)$ diameter and $(d)$ length.

In fact, the observation of the $\mathrm{AO}$ effect in a $2 \mathrm{~mm}$ grating is already a significant result when compared to the same effect in a SF, which requires a length of few centimeters to be noticed. Furthermore, from the simulation results we can observe effects of the different FBG lengths (such as $10 \mathrm{~mm}$ length), allowing optimisation for POF applications that require FBGs with different characteristics such as bandwidth and/or reflected power, among others.

\section{EXPERIMENTAL REALIZATION AND RESULTS}

The Bragg gratings used to perform the experiments were inscribed in PMMA-based few mode mPOF through the phase mask technique, using a continuous wave $\mathrm{HeCd}$ laser with an output wavelength of $325 \mathrm{~nm}$ and a power of $30 \mathrm{~mW}$. The $\mathrm{mPOF}$ has an outer diameter of $150 \mu \mathrm{m}$ and a core diameter of $15 \mu \mathrm{m}$. The core is bounded by four rings of holes as shown in Fig. $9(a)$. Details of the production process of the MPOF are described in [19]. The laser beam was focused vertically downward using a $10-\mathrm{cm}$ focal length cylindrical lens, through a $1061 \mathrm{~nm}$ period phase mask and onto the fiber. The mPOF was laid on a v-groove and taped down using polyimide tape. This type of fiber has a typical inscription time of 40 to 60 $\mathrm{min}$. The Bragg wavelength of the inscribed grating was in the region of $1570 \mathrm{~nm}$ with a length of $2 \mathrm{~mm}$, determined by the width of the UV laser beam. The inscription process was monitored using a $1550 \mathrm{~nm}$ multimode $50 / 125 \mu \mathrm{m}$ silica fiber coupler, a broadband light source, and an optical spectrum analyzer (OSA). For the inscription, a temporary connection was made using a FC/APC contact connector on a 50/125 $\mu \mathrm{m}$ silica fiber which was then butt coupled to the bare POF using an $x-y-z$ translation stage. A small amount of index matching gel was used in the coupling to reduce Fresnel reflections. After the inscription, the grating was spliced to a SF using UV curable glue [20].

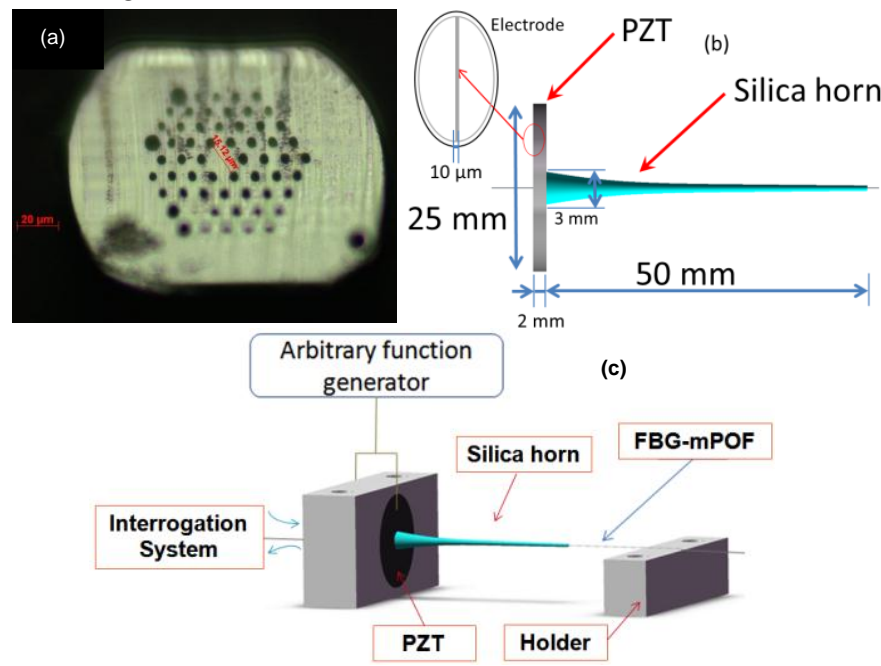


Fig. 9. (a) Microscope image of end face of few mode mPOF. (b) Acoustooptical modulator design and geometric properties. (c) Schematic apparatus used to excite acoustic waves in the silica horn structure.

The AOM is composed of a PZT (PZ26 model disc www.physikinstrumente.com), a silica horn and the mPOF containing the Bragg grating (see Fig. 9 (b)). The AOM is the same used in our previous works [10,11]. The silica horn length is $L_{\text {horn }}=50 \mathrm{~mm}$, with a base diameter $\Phi=3 \mathrm{~mm}$, a tip diameter $\Phi=1 \mathrm{~mm}$ and central hole diameter $\Phi=150.5 \mu \mathrm{m}$. The initial tension of the FBG is adjusted using microresolution translation stages. The end of the fiber is fixed in a holder, which enables the generation of an acoustic standing wave. The acoustic wave interaction length of the horn-fiber is $72 \mathrm{~mm}$ and $80 \mathrm{~mm}$ when the FBG length is $2 \mathrm{~mm}$ and $10 \mathrm{~mm}$, respectively, as shown in the Table 1 . It should be note that the acoustic wave interaction length is the total length of the FBG-AOM, $L_{D}$. The set was swept over the range from $1 \mathrm{kHz}$ to $1.5 \mathrm{MHz}$ to investigate the acoustic effects on the mPOFBG and to compare with FBG in silica fiber. As one can see in Fig. 9 (c), the AOM is connected to an arbitrary function generator and the reflected spectrum was monitored by an OSA with wavelength accuracy of $1 \mathrm{pm}$.

Fig. 10 (a) shows that the acoustic excitation at $77 \mathrm{kHz}$ leads to a broadening of the FBG spectrum, which can be controlled by means of the PZT load $\left(\mathrm{V}_{\mathrm{PZT}}\right)$. When the PZT load is set at 20V, the FBG amplitude is totally suppressed. This result can be explained considering the coupling mechanism of the propagating modes. The lower reflectivity of the grating is due to changing period along the grating length that disrupts the phase matching, leading to a lower coupling to the counter-propagating mode [22]. For example, this behavior can be used for add-drop multiplexers in low frequency regimes. It should be noted that maximum amplitude measurement of each spectrum is made between the values of noise background and main peak (Fig. 10 (a)).

Fig. 10 (b) shows the spectral behavior of the grating when the applied $\mathrm{V}_{\mathrm{PZT}}$ is changed between $0 \mathrm{~V}$ and $20 \mathrm{~V}$ and with the acoustic wave frequency set at $77 \mathrm{kHz}$. It is possible to control the 3-dB bandwidth of the reflected spectrum by tuning the applied $\mathrm{V}_{\text {PZT }}$. As the PZT load is increased the phase mismatching effect also increases. In the case of 3-dB bandwidth measurements, results are presented for PZT loads up to $17 \mathrm{~V}$, the maximum measurable $3-\mathrm{dB}$ bandwidth value. For higher loads, the FBG is suppressed. Figs. 10 (c-e) show the measured-simulated FBG spectra when the grating is excited with the $77 \mathrm{kHz}$ resonance for different $\mathrm{V}_{\text {PZT }}$. The measured spectra are compared to the modelled TMM spectra obtained from previously assessed FEM simulations. At $V_{\mathrm{PZT}}$ $=10 \mathrm{~V}$ the amplitude of the harmonic load delivered by the PZT is $P_{0}=0.63 \mathrm{~N}$ (verified through simulations). The differences in reflectivity and wavelength bandwidth between measured-simulated results are due to grating variations originating in the grating inscription process. The experimental results show a good agreement with the simulated data.
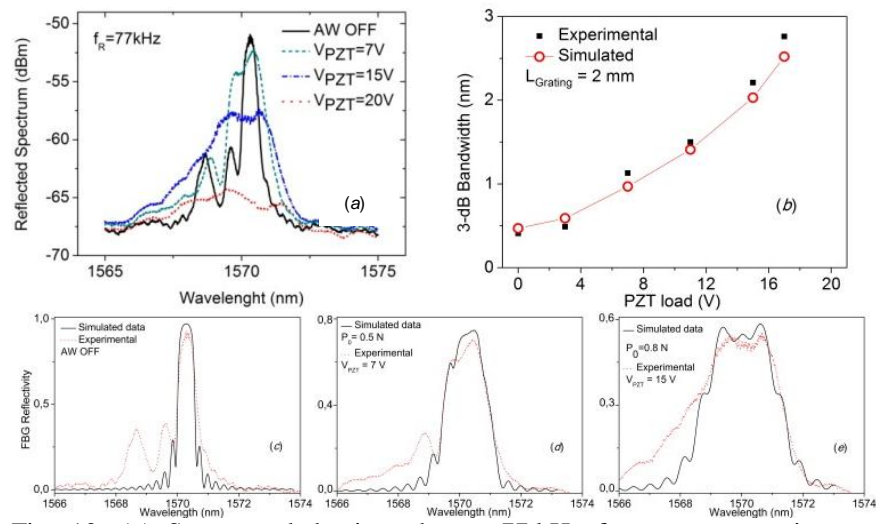

Fig. 10. (a) Spectrum behavior when a $77 \mathrm{kHz}$ frequency acoustic wave excites the mPOFBG, varying $\mathrm{V}_{\mathrm{PZT}}$ from 0 to $20 \mathrm{~V}$. (b) 3-dB FBG bandwidth behavior as a function of the PZT load (experimental results and simulated data) for an acoustic excitation of $77 \mathrm{kHz}$. FBG measured-simulated spectra when (c) AW is OFF, (d) $\mathrm{V}_{\mathrm{PZT}}$ is $7 \mathrm{~V}$ (corresponding an amplitude of the harmonic load $\left.\mathrm{P}_{0}=0.5 \mathrm{~N}\right)$ and $(\mathrm{e}) \mathrm{V}_{\mathrm{PZT}}$ is $15 \mathrm{~V}\left(\mathrm{P}_{0}=0.8 \mathrm{~N}\right)$.

Fig. 11 (a) shows an example of spectral shaping when an acoustic excitation of $193 \mathrm{kHz}$ is turned ON. In this example, the side lobe of the reflection spectrum of the FBG is suppressed, due to an induced apodization, leading to a better filter response. At $193 \mathrm{kHz}$, the acoustic wavelength $(\sim 3.09$ $\mathrm{mm})$ is larger than the grating length $\left(L_{\text {GRATING }}=2 \mathrm{~mm}\right)$. Once the length between the PZT and the beginning of the FBG (60 $\mathrm{mm})$ and the acoustic wavelength are known, the wave position relatively to the FBG can be estimated, i.e. the ratio between them give us the wave position relatively to the FBG as shown in Fig. 11 (b). When acoustic wave is turned ON there is a maximum of the standing acoustic wave at $\mathrm{z}=0$. Taking into account the calculated standing wave period, it is possible to write the FBG in a wave zone which induces an apodization such as the center of the FBG is positioned in a node (zero displacement - see Fig. 11 (b)).

In Fig. 11 (c), the acoustic excitation of $214 \mathrm{kHz}$ results in a spectral broadening of the FBG. This may be a result of the grating being in the transition between the compression and rarefaction strain fields, corresponding to both blue and red shifting of the $\lambda_{\mathrm{B}}$. Therefore, this behavior demonstrates a bandwidth tuning structure as a function of the PZT load.
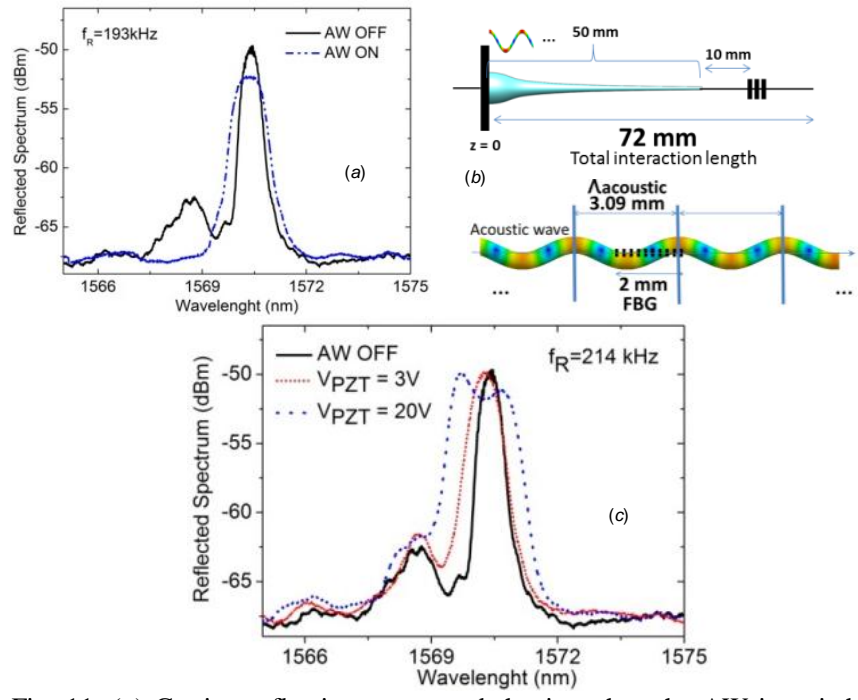

Fig. 11. (a) Grating reflection spectrum behavior when the AW is switched "ON" (20 V) and "OFF" considering a frequency at $193 \mathrm{kHz}$. . (b) Diagram 
showing the wave position relatively to the FBG. (c) Reflected spectrum behavior when the $\mathrm{AW}$ is switched "ON" and "OFF" considering a frequency at $214 \mathrm{kHz}$.

In this experimental work the acoustic excitation is only observed in low-frequency regime, i.e. when the longitudinal acoustic wavelength is longer that the FBG length $(2 \mathrm{~mm})$. Indeed, from Fig. 6 we can conclude that for a device using mPOF, mostly resonance frequencies are located below 1 $\mathrm{MHz}$ (shorter acoustic wavelength). At this particular frequency and for $3.5 \mathrm{MHz}$, the appearance of additional bands on both sides of the grating reflection spectrum should be experimentally observed. Therefore, further developments will be focused in the increasing of the FBG length in order to increase the acoustic-optic interaction length and thus improve the peak reflectivity of the secondary lobules.

\section{CONCLUSION}

In summary, we have demonstrated that the excitation of an FBG written in mPOF through acoustic waves can be attained. Results show that it is possible to dynamically control the properties of the grating spectra even with a grating length of $2 \mathrm{~mm}$ through the AOM, which has not been possible in SFs.

The FEM and the TMM present the advantage of calculating the strain along the whole structure allowing the determination of the influence of the structure dimensions (horn, fiber and FBG sizes) on the grating reflected spectrum when an acoustic wave propagates along its axis. Furthermore, the physical characteristics of the structure can be adjusted in order to obtain the desired strain and FBG spectrum. The approach takes into account the load induced by the acoustic wave, which can be associated with the characteristics of the PZT actuator used for its excitation. The simulation results obtained using the method correlate well with experimental data. The strain field along an mPOFBG-AOM and a similar structure with SFBG-AOM was determined, showing that the strain field amplitude is higher along the mPOFBG due to its smaller mechanical stiffness.

The obtained results can be used in the development of finetuned optical filters using low voltage sources and low frequency regimes, to obtain tunable optical filters and to control the shape of the spectrum. Developments focused on the behavior in different gratings (such as phase shifted FBGs and long period gratings) and different lengths for photonic applications, such as tunable notch filters or tunable cavities, are in progress.

\section{REFERENCES}

[1] H.Yang, S. C. Lee, E. Tangdiongga, C. Okonkwo, H. P. A. van den Boom, F. Breyer, S. Randel, A. M. J. Koonen., " 47.4 Gb/s Transmission Over 100 m Graded-Index POF Based on Rate-Adaptive Discrete Multitone Modulation", J. Lightwave Technol. 28, 352 (2010).

[2] Y.Shao, R. Cao, Y-K. Huang, P. N. Ji, S. Zhang, "112-Gb/s Transmission over 100m of Graded-Index POF for Optical Data Center Applications", OFC'12, OW3J.5 (2012).

[3] W. Yuan, A. Stefani, O. Bang, "Tunable Polymer Fiber Bragg Grating Inscription: Fabrication of Dual-FBG Temperature Compensated Polymer Optical Fiber Strain Sensors", Photon. Techn. Lett. 24, 401 (2012).

[4] W. Zhang, D. J. Webb, and G.-D. Peng, "Investigation Into Time Response of Polymer Fiber Bragg Grating Based Humidity Sensors", J. Lightwave Technol. 30, 1090 (2012).
[5] S.Zhou, L.Reekie, H. P. Chan, Y.T. Chow, P. S.Chung, K. M.Luk, "Characterization and modeling of Bragg gratings written in polymer fiber for use as filters in the THz region", Opt. Express 20, 9564 (2012).

[6] Y.Koike, M. Asai, "The future of plastic optical fiber", NPG Asia Mat. 1, 22 (2009).

[7] H.Dobb, D.J. Webb, "Continuous wave ultraviolet light induced fiber Bragg gratings in few and single-mode microstructured polymer optical fibers", Opt. Letters 30, 3296 (2005).

[8] C.A.F. Marques; R.A. Oliveira; J. Canning, A. Pohl; R.N. Nogueira, "Control of the Properties of Fiber Bragg Gratings Based on the Acousto-Optic Effect," ECOC'10, P1.08 (2010).

[9] G.Fernandes, M. Niehus, C.A.F. Marques, R.N. Nogueira, A.N. Pinto,"Acousto-Optic Tunable Mode Coupler", OFC'12, JTh2A.2 (2012).

[10] C.A.F. Marques, R. A. Oliveira, A. Pohl, R.N. Nogueira, "Tunable Acoustic Bursts for Customized Tapered Fiber Bragg Structures", J. Opt. Soc. Am. B 29, 3367-3370 (2012).

[11] C.A.F. Marques; R.A. Oliveira; A. Pohl; R.N. Nogueira, "Adjustable EDFA Gain Equalization Filter for DWDM Channels Based on a single LPG excited by Flexural Acoustic Waves", Opt. Comm. 285, 3770 (2012).

[12] T.J.R. Hughes, The Finite Element Method - Linear Static and Dynamic Finite Element Analysis, Prentice-Hall, Englewood Cliffs, NJ, (1987).

[13] O.C. Zienkiewicz, R.L. Taylor, The Finite Element Method, vol. 1, The Basis, Oxford, Butterworth-Heinemann, (2000).

[14] H. Kogelnik, in: T. Tamir (Ed.), Theory of Optical Waveguides, in Guided-Wave Optoelectronics, Springer-Verlag, New York, (1990).

[15] K.O. Hill, G. Meltz, "Fiber Bragg Grating Technology Fundamentals and Overview" J. Lightwave Technol. 15, 1263. (1997).

[16] B. Hassan, E. Hinton, "A review of homogenization and topology optimization I-homogenization theory for media with periodic structure", Comput. \& Struct. 69, 707 (1998).

[17] V. V. Vasiliev, E. V. Morozov, Mechanics and Analysis of Composite Materials, Amsterdam: Elsevier (2001).

[18] R.A. Oliveira, P.T. Neves Jr, J. T. Pereira,A. Pohl, "Analysis of Mechanical Properties of a Photonic Crystal FBG Acousto-Optic Modulator", AIP Conf. Proc. 1055, 117 (2008).

[19] G. Barton, M. A. van Eijkelenborg, G. Henry, M. C. J. Large and J. Zagari, "Fabrication of microstructured polymer optical fibres", Opt. Fiber Technol. 10, 325 (2004).

[20] I. Johnson, D. J. Webb, K. Kalli, M.e C. J. Large, A. Argyros, "Multiplexed FBG sensor recorded in multimode microstructured polymer optical fibre", Proc. SPIE 7714, (2010).

[21] R. A. Oliveira, P.T. Neves Jr., J.T. Pereira, A.A.P. Pohl, "Numerical approach for designing a Bragg grating acousto-optic modulator using the finite element and the transfer matrix methods", Opt. Comm. 281, 4899 (2008).

[22] R. A. Oliveira, P.T. Neves Jr., J.T. Pereira, J. Canning, A.A.P. Pohl, "Vibration mode analysis of a silica horn-fiber Bragg grating device", Opt. Comm. 283, 1296 (2010). 